\title{
Digital holography at millimetre wavelengths
}

\author{
Ronan J. Mahon *, J. Anthony Murphy, William Lanigan \\ Department of Experimental Physics, National University of Ireland, Maynooth, Co. Kildare, Ireland
}

Received 8 August 2005; received in revised form 8 November 2005; accepted 9 November 2005

\begin{abstract}
In this paper, we describe a method for wavefront reconstruction at millimetre wavelengths using off-axis holography, a frequently used image recording technique at visible wavelengths first demonstrated in the 1960's. Millimetre radiation has been highlighted recently in the imaging of non-conducting materials and objects, which often show significant transparency at these long wavelengths, even though opaque at visible and infrared wavelengths. Holography provides a method for recording a lens-less image of an object thus reducing loss of spatial frequency information, which is important for obtaining maximum resolution at long wavelengths. An experimental arrangement based on a simple form of Fresnel off-axis holography is described, with the object illumination and reference beams derived using two radiating horn antennas fed by a single coherent source (a Gunn diode oscillator operating at $100 \mathrm{GHz}-\mathrm{wavelength}$ $3 \mathrm{~mm}$ ) via a $3 \mathrm{~dB}$ cross-guide coupler. The process is discussed in the context of imaging and in the validation of millimetre wave radiation sources (horns). We show that the entire fields from these and other components can be analysed using computational optics with data derived from a single measurement obtained using inexpensive equipment.
\end{abstract}

(C) 2005 Elsevier B.V. All rights reserved.

PACS: 07.57.-c; 42.40.- $\mathrm{i}$

Keywords: Millimetre wave; Digital holography; Terahertz imaging

\section{Introduction}

Millimetre-wave electromagnetic radiation bridges the gap between the visible-IR and microwave regions of the spectrum. As in the case of the microwave/radio bands, millimetre-wave propagation tends to be dominated by diffraction effects, since the beam sizes are often of the same order of magnitude as the wavelength. Nevertheless, in a similar way to the infrared and visible bands, it is also possible to guide such beams using relatively compact multi-element optical systems, the design of such systems requiring specialized quasi-optical analysis [2]. Although recent advances in millimetre-wave imaging have often relied on the development of more sensitive detectors, well-developed theories of optical imaging have so far been

\footnotetext{
* Corresponding author. Tel.: +3531708 4668; fax: +35317083313. E-mail address: ronan.j.mahon@nuim.ie (R.J. Mahon).
}

under-exploited to a large extent outside the visible part of the spectrum. Thus there is also the complementary possibility of the novel application of the large range of ingenious optical techniques from the visible band to gain significant expansion in the range of millimetre-wave imaging tools available.

Here we have, for example, developed an imaging system that relies solely on simple models of holography to view the transmission properties of transparent materials. While still in the proof-of-concept stage the images achieved thus far in our investigation show good quality even given the limited size of the objects being examined.

A hologram, in simple terms, is the interference pattern generated by an easily replicable reference beam and the radiation scattered/diffracted by an (unknown) object under investigation, which is then recorded on a photographic plate, although recent advances in CCD technology allow for the digital recording and numerical reconstruction of holograms $[1,3,4]$. This technique of 
digital holography has also enabled wavefront reconstruction using infrared radiation [5], and X-rays [6]. Numerically designed holograms have been employed at the Helsinki University of Technology in millimetre-wave compact antenna test ranges to provide plane waves for farfield beam pattern measurements $[7,8]$. The principles of holography have also been exploited at millimetre wavelengths for use in surveillance for security purposes, but where both amplitude and phase of the scattered light from a target are both detected directly [9]. The work presented in this paper differs from that of [9] in our use of optical techniques in which the phase is not recorded directly but rather it is recovered from a measured interference pattern.

In conventional optical transmission holography illumination of the developed hologram by the reference beam results in the reconstruction of the amplitude and phase variation of the object beam, thus enabling the object beam to be viewed at any plane including its original location.

A hologram is recorded over some plane which we define at $z=0$. The intensity pattern $t(x, y, 0)$ results from interference of the diffuse beam scattered from the object $O(x, y, 0)$ with the plane reference beam $R(x, y, 0)$, so that $t(x, y, 0)=$ $|O(x, y, 0)+R(x, y, 0)|^{2}$. While the intensity terms $|O(x, y, 0)|^{2}$ and $|R(x, y, 0)|^{2}$ vary only slowly with $x$ and $y$, the high frequency fringes or variations in the intensity of the hologram are dominated by the $R^{*}(x, y, 0) \cdot O(x, y, 0)$ and $R(x, y, 0) \cdot O^{*}(x, y, 0)$ interference terms. In fact the hologram acts as a diffraction grating dominated by these interference terms. A virtual image of the object is recovered at its original location from the diffraction order created by the $R^{*}(x, y, 0) \cdot O(x, y, 0)$ term, while the $R(x, y, 0) \cdot O^{*}(x, y, 0)$ term gives rise to a real image in front of the hologram. This reconstruction can also clearly be performed by simulation once the hologram intensity pattern is recorded and the reference beam is accurately described. If a plane wave illuminates the hologram located in the plane $z=0$, the Fresnel-Kirchoff integral results in the complex amplitude $\Gamma(\xi, \eta)$ in the plane, $d$, of the virtual image (where the object was originally located):

$$
\begin{aligned}
\Gamma(\xi, \eta)= & \frac{\mathrm{i}}{\lambda z} \mathrm{e}^{-\mathrm{i} \frac{\pi}{\lambda d}\left(\xi^{2}+\eta^{2}\right)} \iint_{(x, y)} R(x, y) t(x, y) \mathrm{e}^{-\mathrm{i} \frac{\pi}{\lambda d}\left(x^{2}+y^{2}\right)} \\
& \times \mathrm{e}^{\mathrm{i} \frac{2 \pi}{\lambda d}(x \xi+y \eta)} \mathrm{d} x \mathrm{~d} y
\end{aligned}
$$

By performing a fast Fourier transform of the function $R(x, y) t(x, y) \exp \left[-\mathrm{i} \pi\left(x^{2}+y^{2}\right) / \lambda d\right]$ we can recover $\Gamma(\xi, \eta)$, [10]. Although we assume uniform plane wave illumination by the reference beam, the intensity profile of the collimated reference beam is actually Gaussian though with a large extent at the hologram. Over the small region scanned in recording the hologram, the reference beam amplitude varies only slowly and the large intensity fluctuations are dominated by the pattern of interference fringes produced in the presence of the illuminated object beam. We could therefore approximate the reference beam through its phase front only, which can be considered as planar (as the reference Gaussian beam has a waist at the recording plane produced by the paraboloidal mirror as in Fig. 1), i.e., $R(x, y, 0)=\exp \left(\mathrm{i}\left(k_{x} x+k_{y} y\right)\right)$, where $\left(k_{x}, k_{y}\right)$ are the direction cosines for the reference beam wavevector. In terms of Eq. (1) given above, $(\xi, \eta)$ represents the point's off-axis location at the plane where the object is located.

\section{Experimental procedure}

A $3 \mathrm{~dB}$ cross waveguide coupler was attached to the transmitting chain of a $100 \mathrm{GHz}$ Gunn diode oscillator source thus eliminating the requirement for beam-splitter in the optical setup, which allowed for a more compact system. A $3 \mathrm{~dB}$ cross-guide coupler is effectively an optical 2-channel multiplexing waveguide component, dividing the total Gunn output of $35 \mathrm{~mW}$ into two beams with equal powers. The reference beam was derived by placing the predicted phase centre of a conical corrugated horn at the focal plane of a parabolic mirror, which collimated the reflected beam with an angle of incidence of $45^{\circ}$ to the hologram plane. The quantity

$\frac{I_{\max }-I_{\min }}{I_{\max }+I_{\min }}$

of an interference pattern $I$ defines its contrast (fringe visibility), which, in holography, plays an important role in the quality of the reconstructed images. In the technique described here, a maximum contrast of unity is easily obtained through the adjusting of signal attenuators attached to the object and reference beam sources.

Holograms were recorded using a scanning detector, with the centre of each hologram lying directly in front of the scattering/diffracting object to be imaged. Care was required to ensure that the reference beam was not vignetted

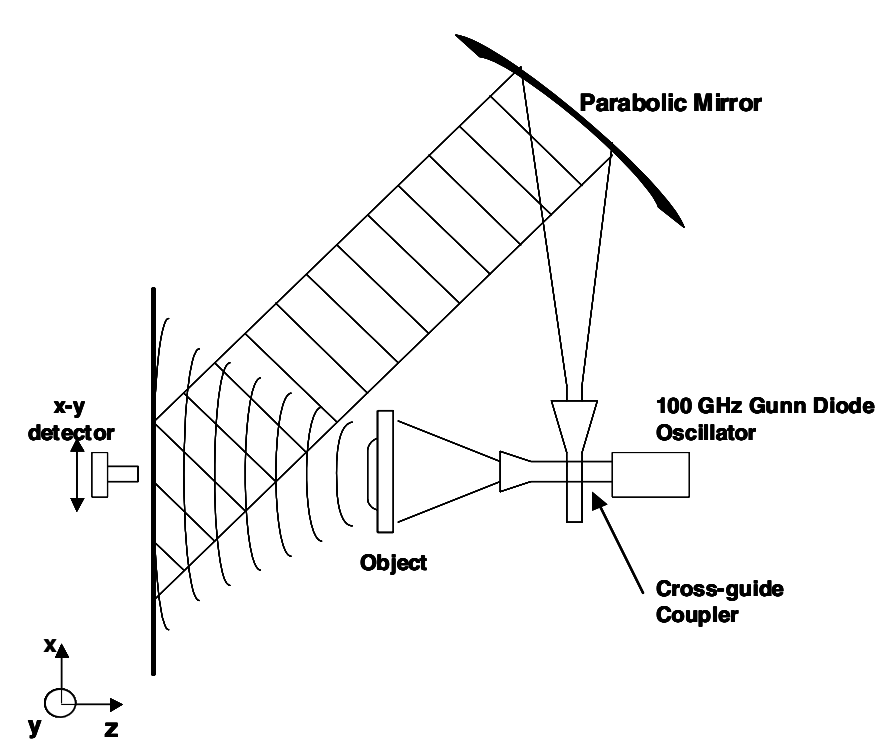

Fig. 1. Experimental arrangement for the creation of digital millimetrewave holograms. The system has an area of approximately $600 \times 600 \mathrm{~mm}^{2}$, with holograms recorded over an area of $\sim 150 \times 150 \mathrm{~mm}^{2}$. 
by the object, which would produce edge diffraction effects contaminating the desired reference wavefront. Instead of employing a set of diverging optics, which is used in transmission holography at visible wavelengths to gain maximum illumination of the object, a horn with a large flare angle, and thus a rapidly diverging beam, is used. A schematic diagram of the experiment is shown in Fig. 1.

At the hologram plane, a beveled untapered waveguide directly feeds a Schottky-barrier diode that acts as a total power detector. The waveguide protrudes through a thin section of Eccosorb ${ }^{\mathrm{TM}}$, which is used at these frequencies to prevent large unwanted reflections from conducting surfaces in quasi-optical systems. These can cause standing waves [11], thereby creating a permanent artifact on the measured hologram that would reduce the quality of the image reconstruction.

The simplest demonstration of holography is where the object is a point-like source (size of order of the wavelength). As an example we can consider the interference pattern produced by the arrangement when the object shown in Fig. 1 is removed and the object illumination beam produced by a corrugated conical horn directly illuminates the recording plane. Unlike coherent sources in the visible regime, beams derived from narrow-band millimetre wave sources exhibit extreme diffraction with the variation in beam width, or waist, $W$, given by $[2,12]$,

$$
W(z)=\sqrt{w_{0}^{2}\left[1+\left(\frac{\lambda z}{\pi w_{0}^{2}}\right)^{2}\right]}
$$

where $w_{0}$ is the waist radius at the source, which in this case is small enough to be considered similar to a quasi-point source. In the experimental arrangement the horn was located $550 \mathrm{~mm}$ from the plane where the hologram pattern was recorded.

To provide another example of wavefront reconstruction a hologram of two pyramidal horns fed by the same source was recorded. The horns, with diameters of $26 \mathrm{~mm}$, were separated by $90 \mathrm{~mm}$ in the vertical plane and located $500 \mathrm{~mm}$ from the plane of the hologram. In this case interference should occur between both object beams, and visualisation of this is sought in the digital reconstruction.

We also consider the case where a screen with a complex shaped aperture truncates the object illumination beam. A slightly irregular ' $M$ ' shaped mask was cut from Eccosorb ${ }^{\mathrm{TM}}$ and mounted on a sheet of polystyrene foam, which at terahertz frequencies has a refractive index simi- lar to that of air [13]. The object used has a total area of $60 \times 60 \mathrm{~mm}^{2}$, with its smallest feature having an extent of $9 \mathrm{~mm}$.

\section{Results}

Fig. 2 shows the hologram for the first arrangement described above. In this instance the holograms fringe structure was predicted, using a point source approximation, to be of the form:

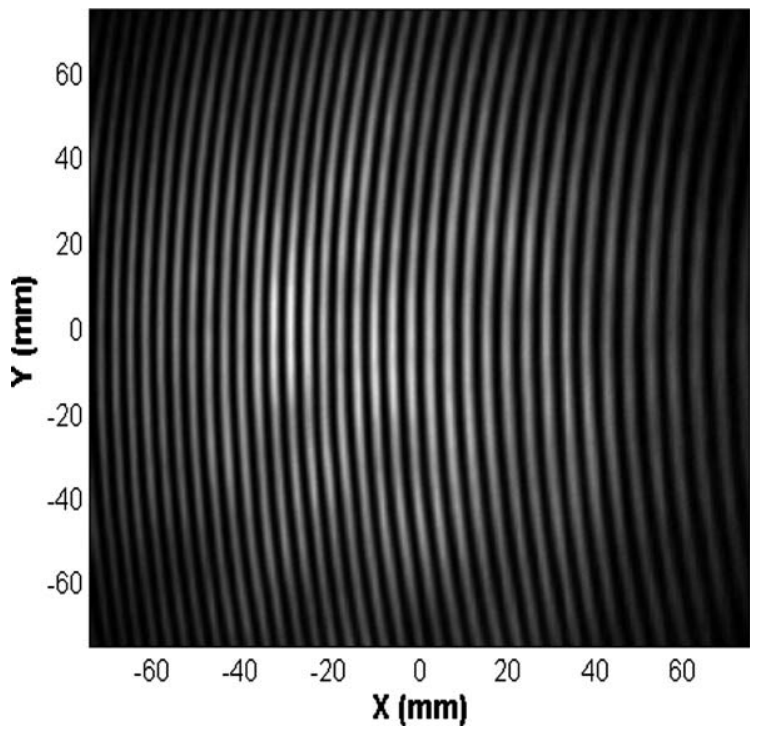

Fig. 2. Recorded hologram of a compact radiating horn (aperture diameter of $14.5 \mathrm{~mm})$.

$t(x, y, 0) \propto\left|\exp \left(\mathrm{i} \frac{k\left(x^{2}+y^{2}\right)}{2 d}\right)+\exp \left(\mathrm{i} k_{x} x+\mathrm{i} k_{y} y\right)\right|^{2}$

where $k_{x}=2 \pi \sin \theta_{x} / \lambda$, and $\theta_{x}$ is the angle of incidence of the reference beam with respect to a normal of the hologram plane. We assume that the beam has no angular phase component in the vertical plane, i.e. $k_{y}=0$.

Upon comparison of Fig. 2 with the predicted point source hologram as given by (4), the recorded hologram shows excellent agreement in terms of fringe periodicities and curvatures. Thus, the field from a horn having dimensions of a similar order of magnitude as the wavelength may be indistinguishable from the field generated by a point source, hinting at limitations in this form of imaging at long wavelengths.

Using the simple model described in Eq. (1), diffraction software was developed to calculate the field at various planes in the image field, allowing for the analysis of millimetre wave sources. Fig. 3 shows such a reconstruction, exhibiting the variation in beam width described by Eq. (3).

In the very-near field of the hologram one can see fringes formed by the interference of the object beam with the reference beam. Because we assume a reference beam having a horizontal wavevector i.e., $k_{y}=0$, the reconstruction mimics the superposition of a plane wave with the quasi-spherical beam from the object, thus allowing for the examination of the wavefront (see Fig. 4).

The reconstruction from the hologram of two radiating horns also shows the entire field in front of the hologram, and as with the previous example, the curvature of the object field near the hologram can be seen through interference with the reference field, showing the overlapping spherical wavefronts' of both object beams. This is a useful result for the testing of lens antennas and horns. 


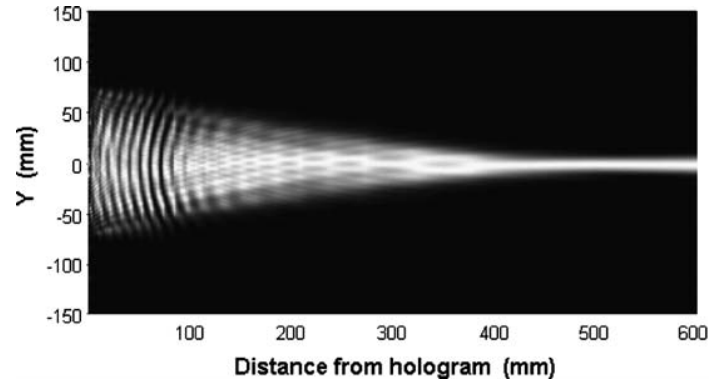

Fig. 3. Reconstruction of hologram in Fig. 2. The source was located at $550 \mathrm{~mm}$ from the hologram. Note scale is linear and the beam is normalised on-axis to unity at all planes.

a
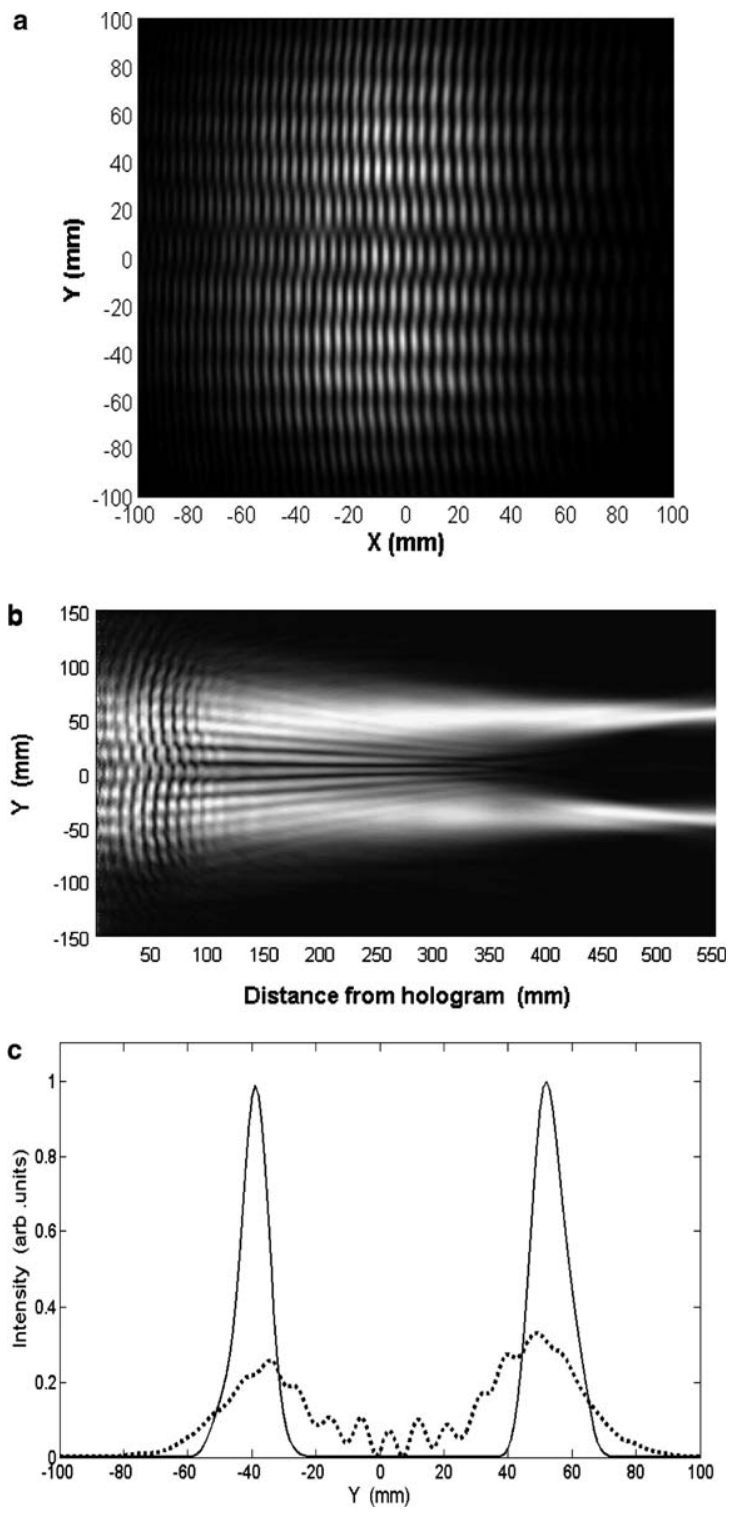

Fig. 4. (a) Hologram of two vertically arranged horns; (b) reconstructed field (vertical cut) and (c) Object field at $250 \mathrm{~mm}$ (dotted line) and its original location $500 \mathrm{~mm}$ (solid line) from hologram plane.

Fig. 5 shows the hologram and reconstructed image of an Eccosorb ${ }^{\mathrm{TM}}$ mask. The dimensions of the aperture as quoted in the previous section are of a similar order of
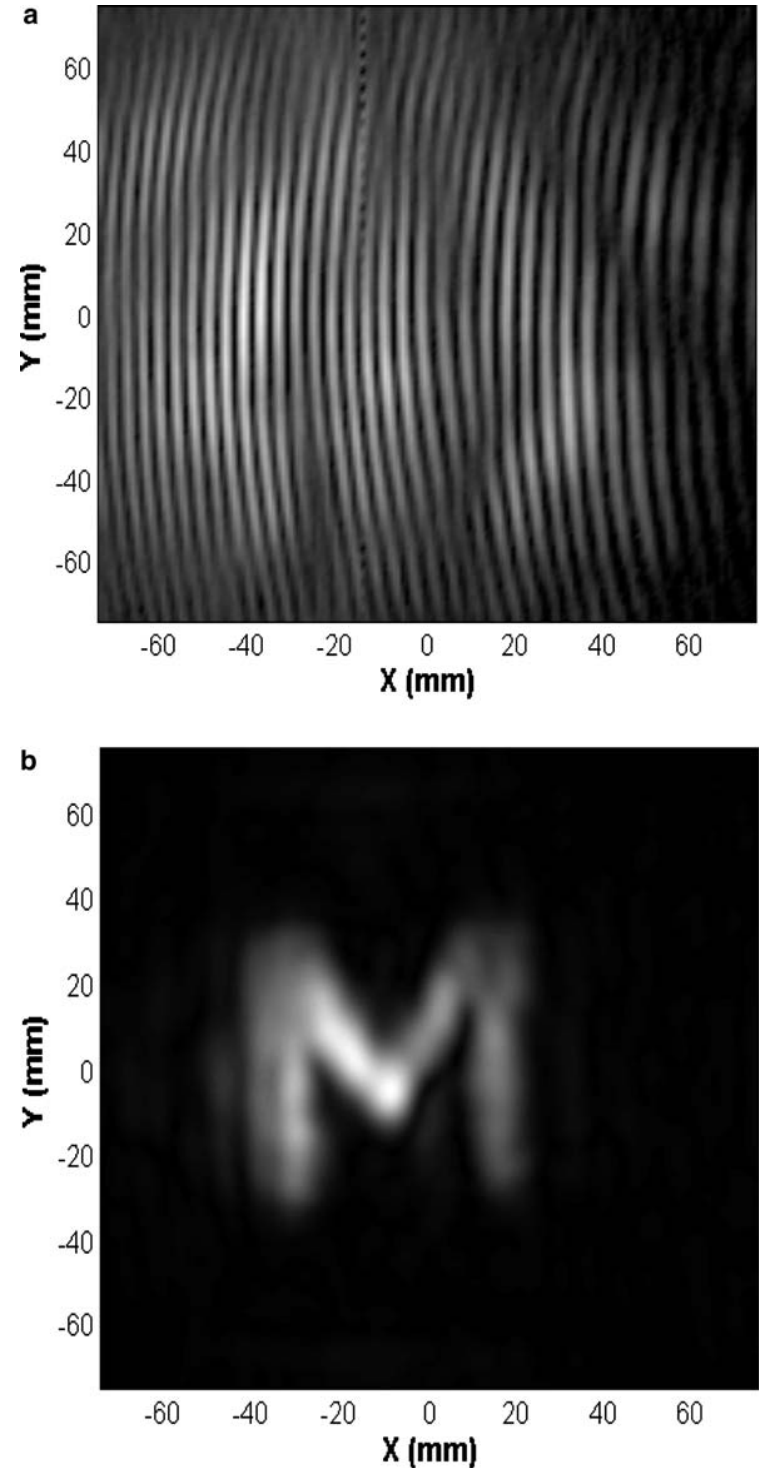

Fig. 5. (a) Hologram of ' $M$ ' shaped mask and (b) digital reconstruction at objects original location.

magnitude to that of wavelength, showing that the lensless imaging performed by holography can provide high-resolution imaging at these wavelengths.

\section{Conclusion}

We have demonstrated the use of numerical holographic image reconstruction at millimetre wavelengths using a straightforward and compact system with possible applications in the determination of the transmission characteristics for non-conducting materials and objects. The ability to calculate phase and amplitude information from a single experiment shows promise in the analysis of millimetrewave receiver horns, particularly the determination of phase centres, which is important for optimizing resolution on astronomical telescopes. 
The evaluation of optical components at millimetrewave and terahertz frequencies can be very time consuming and often requires the measurement of diffracted or reflected fields at several planes. Here, we have shown how from one experimental measurement, numerical techniques can be used to reconstruct the entire output field from a linear optical arrangement.

In the context of imaging, the results obtained show the possibility of recovering high resolution for relatively small objects with dimensions of the order of $\sim 10 \lambda$ in size. The development of a similar system that would allow for the holographic imaging of scattering or reflective objects is being planned. This will have applications in a study of the absorption and reflection properties of organic and bio-medical samples at $100 \mathrm{GHz}$, and work is ongoing in the development of an extension of this experiment in such a direction.

It is worthwhile commenting that the optical processes involved in holography, such as the formation of interference fringes, are often more easily demonstrated using longer wavelengths than with optical wavelengths. This is due to the low sampling rate required to recover such fringes (for example at optical wavelengths such fringes are more difficult to recover with conventional CCD recording systems). Thus at long wavelengths it is often feasible to demonstrate the operation of novel optical schemes (especially those relying on phase modulation of beams), proving millimetre wavelength radiation to be as useful in the study of classical and modern optical phenomena as visible light in such recent topics as Bessel beams and optical vortices [14-16].

Recent papers have suggested the application of holography in $\mathrm{THz}$ imaging using the high-power radiation produced by free-electron lasers $[17,18]$. The power levels of such sources (up to $600 \mathrm{~W}$ ) could enable more sophisticated medical imaging of highly absorbing organic tissue to be realised.

Holography in the visible band has provided hundreds of applications, either obvious imaging modalities, or more abstract information and signal processing techniques. With the rapid expansion of technology applicable to terahertz and millimetre wave photonics, it is difficult to see how holography cannot provide several further techniques to these fields.

\section{Acknowledgement}

This work is supported financially by the Irish Research Council for Science, Engineering, and Technology.

\section{References}

[1] E.N. Leith, J. Upatneiks, Journal of the Optical Society of America 54 (1965) 1295.

[2] P. Goldsmith, Quasi-Optical Systems: Gaussian Beam Propagation and Applications, IEEE Press, 1998

[3] U. Schnars, W. Juptner, Applied Optics 33 (1994) 179.

[4] T.J. Naughton, Y. Frauel, B. Javidi, E. Tajahuerce, Applied Optics 41 (20) (2002) 4124.

[5] E. Allaria, S. Brugioni, S. De Nicola, P. Ferraro, S. Grilli, R. Meucci, Optics Communications 215 (4-6,15) (2003) 257.

[6] S. Eisebitt, J. Lüning, W.F. Schlotter, M. Lörgen, O. Hellwig, W. Eberhardt, J. Stöhr, Nature 432 (2004) 885.

[7] J. Meltaus, J. Salo, E. Noponen, M.M. Salomaa, V. Viikari, A. Lönnqvist, T. Koskinen, J. Säily, J. Häkli, J. Ala-Laurinaho, J. Mallat, A.V. Räisänen, IEEE Transactions on Microwave Theory and Techniques 51 (4) (2003).

[8] A. Lonnqvist, T. Koskinen, J. Häkli, J. Saily, J. Ala-Laurinaho, J. Mallat, V. Viikari, J. Tuovinen, A.V. Raisanen, Antennas and Propagation IEEE Transactions on 53 (10) (2005) 3151.

[9] D.L. McMakin, D.M. Sheen, H.D. Collins, 30th Annual International Carnahan Conference (1996) 19.

[10] J.W. Goodman, Introduction to fourier optics, second ed., McGraw Hill, 1996.

[11] J.A. Murphy, N. Trappe, S. Withington, Infrared Physics and Technology 44 (2003) 289.

[12] J.A. Murphy, A. Egan, European Journal of Physics 14 (1993) 121.

[13] G. Zhao, M. ter Mors, T. Wenckebach, P.C.M. Planken, Journal of the Optical Society of America B 19 (6) (2002) 1476.

[14] G.A. Turnbull, D.A. Robertson, G.M. Smith, L. Allen, M.J. Padgett, Optics Communications 127 (1996) 183.

[15] N. Trappe, R. Mahon, W. Lanigan, J.A. Murphy, S. Withington, Infrared Physics \& Technology 46 (3) (2005) 233.

[16] J. Courtial, D.A. Robertson, K. Dholakia, L. Allen, M.J. Padgett, Physical Review Letters 81 (1998) 4828.

[17] V.S. Cherkassky et al., Nuclear Instruments and Methods in Physics Research A 543 (2005) 102.

[18] Y.U. Jeong et al., Nuclear Instruments and Methods in Physics Research A 543 (2005) 90. 